\title{
MODELAGEM E SIMULAÇÃO DE UM AVIÁRIO EM UM MEIO POROSO - ANÁLISE DAS CONDIÇÕES DE CONFORTO PARA AS AVES
}

\author{
Mayara Batista1, Ivo Neitzel2* \\ 1 - Faculdade de Telêmaco Borba - FATEB - PR \\ 2 - Faculdade de Telêmaco Borba - FATEB - PR, ivo.neitzel@fatebtb.edu.br
}

\begin{abstract}
Quantificar parâmetros para a qualidade de vida em um aviário, por meio de simulações, é indubitavelmente uma tarefa complexa. A velocidade do ar, a temperatura e concentração de contaminantes são parâmetros importantes, tem um impacto muito grande no crescimento e taxa de mortandade das aves. Para conduzir estes parâmetros a valores adequados e realistas, optou - se por simulações estratégicas a partir de um meio poroso com uma matriz sólida constituída pelas aves, a região das aves $(\boldsymbol{R} \boldsymbol{A})$. A modelagem preconizada possibilitou analisar o impacto causado pela resistência do ar, provocada pela movimentação das aves sobre o escoamento do fluido, transporte de massa e energia. Os resultados obtidos ficaram dentro dos parâmetros estabelecidos para garantir o conforto térmico e a sobrevivência das aves dentro do aviário.
\end{abstract}

Palavras-chave: avicultura; conforto térmico; simulação.

\section{INTRODUÇÃO}

A análise, por simulação, do ambiente interno de aviários é importante para um projeto adequado que preserve as condições de conforto das aves [1]. O aviário, sob este enfoque, pode ser caracterizado pelas seguintes partes:

1. Região da cama do aviário (RC): região que contém a alimentação, a coleta de dejetos das aves, penas e outros materiais cuja finalidade é atenuar efeitos indesejáveis para as aves [2]. Fica ao nível do solo. Gera gases potencialmente poluentes.

2. Região das aves (RA): contém o espaço ocupado pelo corpo principal das aves. Fica a $5 \mathrm{~cm}$ do solo e se estende até $30 \mathrm{~cm}$ (dimensões aproximadas, alteráveis de acordo com a idade e tamanho das aves). Gera calor. Nesta região o ar circula restrito pelas aves.

3. Região livre (RL): região de livre circulação do ar ocupa o espaço acima das aves até o teto do aviário. Pode conter ventiladores internos para circulação do ar interno.

4. Ventiladores externos: responsáveis pela renovação do ar. Podem introduzir ou remover ar.

5. Janela de admissão ou exaustação: dependendo da funcionalidade dos ventiladores externos, o ar entra ou sai por estes orifícios.

6. Eventuais fontes ou sumidouros de calor e umidade.

A região das aves é a que deve ser criteriosamente investigada em relação aos parâmetros de conforto das aves: velocidades limítrofes, temperaturas máxima e mínima e concentrações de amônia $\left(\mathrm{NH}_{3}\right)$. Os valores para essas variáveis estão relacionados de acordo com a idade das aves [1]. Nas primeiras semanas de vida, para a temperatura são recomendados valores próximos de $35^{\circ} \mathrm{C}$, e para aves próximas ao abate, temperaturas amenas, próximas de $20 \mathrm{e}$ $26^{\circ} \mathrm{C}$ [3]. Já a velocidade ideal varia de $0,1 \mathrm{a} 0,7 \mathrm{~m} / \mathrm{s}$ para os pintainhos e a partir da quarta semana até o abate, os valores chegam até $3,5 \mathrm{~m} / \mathrm{s}$ sem restrição [4]. Para a amônia o limite máximo de exposição é de 20 ppm [5].

Sob o enfoque da movimentação do ar, sua espessura ocupa tipicamente $10 \%$ da altura do aviário e fica um pouco acima (em relação ao solo) da espessura da camada limite hidrodinâmica, 3 - 5 \% como referência. As aves, com densidades ocupacionais na ordem de 14 aves por $\mathrm{m}^{2}$ [6], são obstáculo à livre circulação do ar. A ocupação volumétrica pelas aves, nesta região, é de aproximadamente $20-40 \%$. As aves se movimentam randomicamente e, em decorrência das penas, tem superfície não lisa gerando resistência à 
movimentação do ar. $\mathrm{O}$ ar é alimentado a temperatura ambiente $20^{\circ} \mathrm{C}$.

A descrição do comportamento fluidodinâmico e térmico da região $(\boldsymbol{R} \boldsymbol{A})$ próxima ao solo (que tem impacto no transporte de espécies por convecção e difusão dos gases produzidos na cama) foi feita por um modelo que considera a resistência provocada pelas aves. A descrição considera esta região como um meio poroso com uma matriz sólida constituída pelas aves. A porosidade, onde é a fração ocupada pelas aves, considera-as como tendo forma esférica com volume equivalente definido pela sua densidade mássica. A área superficial é calculada a partir da área destas esferas. A parte vazia foi considerada como sendo constituída pelos poros.

\section{METODOLOGIA}

\section{ESCOAMENTO EM MEIOS POROSOS}

A descrição clássica para o escoamento de líquidos e gases em meios porosos com escoamento monofásico e laminar utiliza a Lei de Darcy [7] juntamente com a equação da conservação de massa:

$$
\begin{aligned}
& \frac{\partial\left(\varepsilon_{L} \cdot \rho\right)}{\partial t}=-\left(\nabla \bullet \rho \cdot \mathbf{v}_{\mathbf{0}}\right) \\
& \nabla \wp=-\frac{\mu}{\kappa} \cdot \mathbf{v}_{\mathbf{0}}, \nabla \wp=\nabla p-\rho \cdot \mathbf{g}, \text { ou, } \\
& \mathbf{v}_{\mathbf{0}}=-\frac{\kappa}{\mu} \cdot \nabla \wp \quad \text { (lei de Darcy). }
\end{aligned}
$$

Este modelo foi modificado por Brinkman para considerar a distorção provocada por efeitos viscosos no perfil de velocidade [7] pela proximidade das paredes por intermédio do termo $\nabla^{2} \mathbf{v}_{\mathbf{0}}[8,9]:$

$$
\begin{aligned}
& \nabla \wp=-\frac{\mu}{\kappa} \cdot \mathbf{v}_{\mathbf{0}}+\mu_{e f f} \cdot \nabla^{2} \mathbf{v}_{\mathbf{0}}, \text { ou, } \\
& \mathbf{v}_{\mathbf{0}}=-\frac{\kappa}{\mu} \cdot \nabla \wp+\kappa \cdot \frac{\mu_{e f f}}{\mu} \cdot \nabla^{2} \mathbf{v}_{\mathbf{0}}
\end{aligned}
$$

Nesta equação $\mu_{\text {eff }}$ é uma viscosidade efetiva que descreve a viscosidade na camada limite na superfície da matriz porosa [10] e pode ser diferente de $\mu$ [11]. Na sua essência a correção de Brinkman interfere no escoamento próximo à superfície sólida, produzindo uma redução mais suave da velocidade até o valor zero [10]. Para diâmetros de poros muito pequenos a variação de velocidade através dos poros pode ser desprezada [9]. Para porosidades maiores, e, neste contexto, esta parcela não é considerada.

Para escoamentos com velocidades maiores, $3>\operatorname{Re}>0.1$ [9], Forchheimer postulou [12, 13, 14], por analogia com escoamentos em tubos, a equação (na sua forma original, unidimensional): 


$$
-\frac{\Delta p}{\Delta x}=\frac{\mu}{\kappa} \cdot v_{0}+b \cdot \rho \cdot v_{0}^{m}
$$

O coeficiente da equação de Forcheimer usualmente é colocado na forma, $b=\alpha / k^{\beta}$, [15]. Esta equação, também desenvolvida teoricamente, [16], foi posteriormente modificada por Ergun com base em medições experimentais para a forma atual (existem pequenas variações na forma de escrita desta equação):

$$
\nabla \wp=-\frac{\mu}{\kappa} \cdot \mathbf{v}_{\mathbf{0}}-F_{\text {Forchheimer }} \cdot \frac{\rho}{\sqrt{\kappa}} \cdot\left|\mathbf{v}_{\mathbf{0}}\right| \cdot \mathbf{v}_{\mathbf{0}}
$$

Ela pode, também, ser obtida, pela soma de duas parcelas desenvolvidas especificamente, uma para o regime laminar e outra para o regime turbulento [17], produzindo a forma denominada de equação de Ergun:

$$
-\frac{\Delta \wp}{\Delta x}=\underbrace{\frac{A \cdot \mu}{d_{\text {eff }}^{2}} \cdot\left[\frac{\left(1-\varepsilon_{L}\right)^{2}}{\varepsilon_{L}^{3}}\right]}_{\text {parcela-laminar, Blake-Kozeny }} \cdot v_{0}+\underbrace{\frac{B \cdot \rho_{L}}{d_{\text {eff }}} \cdot\left[\frac{\left(1-\varepsilon_{L}\right)}{\varepsilon_{L}^{3}}\right] \cdot v_{0}^{2}}_{\text {parcela - turbulenta, Burke-Plummer }}
$$

Os valores para as constantes $A$ e $B$ são $A=150$ ou $A=180$ e $B=1.75 \sim 4$, [18]. Nestas equações a velocidade superficial do fluido, $v_{0}$, é definida, [6], como sendo a "vazão mássica dividida pela massa específica" dividida pela "área da coluna na ausência de recheio". A velocidade média com que o fluido escoa pelos espaços vazios é $v_{i}=v_{0} / \varepsilon_{L} ; \varepsilon_{L}$ é a fração de vazios do leito. $d_{\text {eff }}$ é o diâmetro efetivo, que, se as partículas do leito fossem esferas iguais, seria o diâmetro da esfera. Em não sendo este o caso, $d_{\text {eff }}=6 \cdot V / A[10]$. Comparando a equação (7) com a equação (8), verifica-se que:

$$
\begin{gathered}
\kappa=\frac{d_{\text {eff }}^{2} \cdot \varepsilon_{L}^{3}}{A \cdot\left(1-\varepsilon_{L}\right)^{2}} \\
F_{\text {Forchheimer }}=\frac{B}{\sqrt{A \cdot \varepsilon_{L}^{3}}}
\end{gathered}
$$

$$
\kappa=\frac{d_{\text {eff }}^{2} \cdot \sqrt{\varepsilon_{L}^{3}}}{F_{\text {Forchheimer }} \cdot\left(1-\varepsilon_{L}\right)^{2}} \cdot \frac{B}{\sqrt{A^{3}}}
$$

Onde,

$\varepsilon$ é a porosidade do leito (fração de vazios);

$k$ é a permeabilidade do leito;

$v_{0}$ é a velocidade superficial do leito;

$q$ é a vazão volumétrica através da seção reta da "torre";

$A$ é a área da seção reta, perpendicular à direção de escoamento; 
$L$ é o comprimento do leito poroso na direção do escoamento;

$\Delta p$ é a diferença de pressão através do leito (comprimento $L$ );

$\mu$ é a viscosidade do fluído;

$k$ é a permeabilidade, considerada uma propriedade material do meio poroso.

$F_{o}=F_{\text {Forchheimer }} \cdot \frac{\rho \cdot v_{0} \cdot \sqrt{k}}{\mu}$, número de Forchheimer.

A equação (8) é deduzida presumindo que não existam escoamentos preferenciais no leito [6], condição que no presente caso certamente não é respeitada.

A geração de calor é descrita pelo modelo

$\rho c_{p} \mathbf{v} \cdot \nabla T+\nabla \cdot \mathbf{q}=Q_{\text {fonte }}, q=k \cdot \nabla T$

A geração de amônia é descrita pelo modelo de [19]

$\left\langle\mathrm{NH}_{3} \mathrm{ER}\right\rangle=0.32 \pm 0.1 \mathrm{~g} / \mathrm{bird} \cdot \mathrm{day}$

$\nabla \cdot T_{i}+\mathbf{u} \cdot \nabla c_{i}=S_{i},-D_{e j} \nabla c_{i}+\mathbf{u} c_{i}$

\section{SELEÇÃO DE UM MODELO DE ESCOAMENTO PARA A REGIÃO DAS AVES}

Face à dificuldade de realizar um experimento real para validar a modelagem proposta, foram conduzidas simulações nas condições preconizadas na descrição da estratégia de modelagem. A simulação foi realizada com o programa COMSOL 5.2 $2^{\mathrm{a}}$ utilizando o módulo de CFD (Fluidodinâmica Computacional).

Cada ave é representada por uma esfera cujo raio é calculado assumindo que a massa da ave está contida inteiramente na esfera, denominada de esfera equivalente. $O$ inverso da densidade ocupacional de aves é a área específica das aves e o quadrado com a mesma área foi denominado de área de conforto. $\mathrm{O}$ volume de conforto disponível para uma ave foi definido como sendo o cubo cujo lado é igual ao lado do quadrado da área de conforto. As condições e valores paramétricos utilizados estão na Tabela 1.

\begin{tabular}{lll}
\hline Variáveis & Valores de Referência & Unidade \\
\hline Densidade das aves & 400 & $\mathrm{~kg} / \mathrm{m}^{3}$ \\
\hline Volume da esfera equivalente (uma ave) & 2.5 & $\mathrm{~L}$ \\
\hline Raio da esfera equivalente & 0.084 & $\mathrm{M}$ \\
\hline Densidade de ocupação pelas aves & 12 & $\mathrm{aves} / \mathrm{m}^{2}$ piso \\
\hline $\begin{array}{l}\text { Massa de uma ave } \\
\text { Comprimento do lado área de conforto }\end{array}$ & 1 & $\mathrm{Kg}$ \\
\hline $\begin{array}{l}\text { Volume específico da região das aves } \\
\text { (volume de conforto / } 1 \mathrm{~m}^{2} \text { de piso) }\end{array}$ & 0.28875 & $\mathrm{M}$ \\
\hline $\begin{array}{l}\text { Volume ocupado pelas aves (fração } \\
\text { ocupada) }\end{array}$ & 0.1782 & $\mathrm{~m}^{3} / \mathrm{m}^{2}$ \\
\hline
\end{tabular}




\begin{tabular}{lll}
\hline Fração de vazios & 0.8218 & \\
\hline Fração superfície das esferas por $\mathrm{m}^{2}$ de piso & 1.0689 & $\mathrm{~m}^{2} / \mathrm{m}^{2}$ piso
\end{tabular}

Tabela 1 - Valores paramétricos utilizados na simulação

Foi construído um sistema com múltiplos volumes de conforto contendo, cada um, uma esfera equivalente cujo centro coincide com o centro do cubo do volume de conforto. A figura 1 exemplifica este sistema.

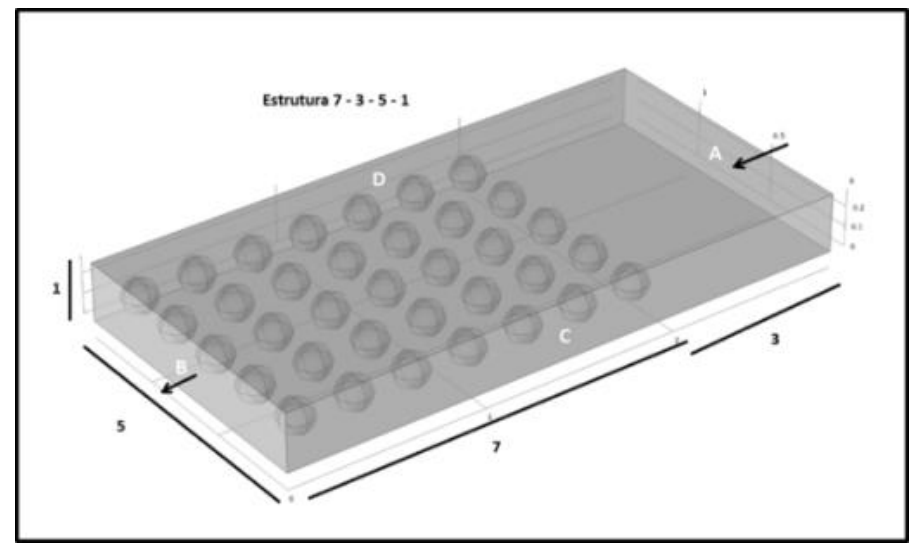

Fig. 1 - Sistema com múltiplos volumes de conforto

Na situação simulada o ar entra pela superfície A com velocidade $v A$ e sai pela face $\mathrm{B}$. A figura se estende, simetricamente, pelas faces C e D. Na estrutura da figura existem 7 esferas equivalentes na direção x, 5 na direção y e 1 na direção z. Três (3) volumes "vazios" precedem o conjunto de esferas. Esta estrutura foi denominada de 7-3-5-1.

\section{RESULTADOS E DISCUSSÃO}

A partir da modelagem preconizada, foram simulados quatro aviários com diferentes arranjos físicos. Os parâmetros a ser avaliados é o impacto causado sobre a temperatura, velocidade do ar e a concentração de $\mathrm{NH}_{3}$. Os aviários foram denominados v701, v702, v703 e v704. Possuem uma área de $525 \mathrm{~m}^{2}$, composto de ventiladores exaustores e uma janela de admissão de ar. O comportamento pressão - vazão é calculada pelo sistema. De fato, o que muda nos arranjos físicos adotados, é a posição dos ventiladores exaustores e a posição da janela de admissão de ar. As versões 701 e 702 possuem duas paredes posicionas de modo a facilitar a circulação do ar na região das aves $(\boldsymbol{R} \boldsymbol{A})$, região de domínio do estudo.

Analisando o comportamento fluidodinâmico e térmico da $\boldsymbol{R} \boldsymbol{A}$, para a temperatura, os ambientes mostraram - se favoráveis para o desenvolvimento de aves próximo ao abate, no qual a temperatura varia de 20 a $26 \mathrm{C}^{\circ}$. Nota - se que não houve grande impacto na temperatura quanto à posição dos ventiladores, a diferença maior é constatada quando comparado os arranjos físicos adotados entre os dois primeiros e os dois últimos ambientes simulados, prevalecendo uma temperatura maior nos aviários v703 e v704, entretanto os resultados são significativos e considerados dentro dos parâmetros de conforto estabelecidos. O comportamento do perfil de temperatura dos aviários é mostrado na figura 3. 


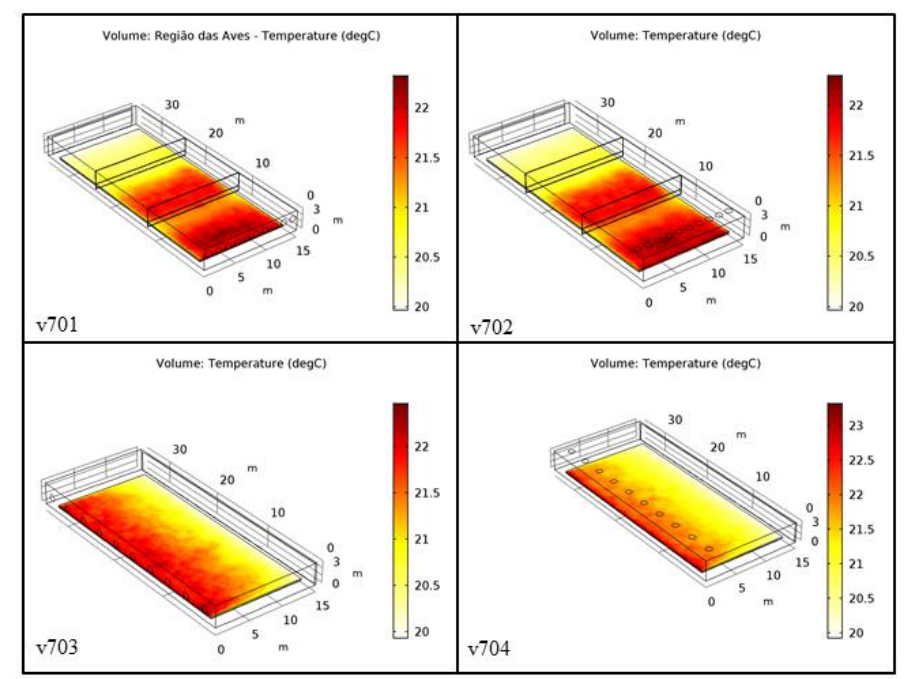

Fig. 3 - Perfil de temperatura na $\boldsymbol{R A}$

A escolha do arranjo físico é sem dúvida essencial para garantir o conforto térmico às aves, quando o fluxo de ar entra no sentido longitudinal, percorre um espaço muito maior que no sentido transversal. Nos aviários v701 e v702, as paredes dispostas no interior do aviário mostraram sua importância, pois forçaram o ar passar homogeneamente na região das aves, aumentando a velocidade do ar, resultando em temperaturas favoráveis ao desenvolvimento das aves. Para os aviários v703 e v704, a diferença foi a direção do fluxo, no sentido transversal, não foi necessário utilizar barreiras, o que explica a baixa velocidade com temperaturas dentro dos limiteis aceitáveis, o percurso para renovação do ar é significativamente menor, o que facilita a troca de ar no interior dos galpões. Conforme é apresentado na figura 4.

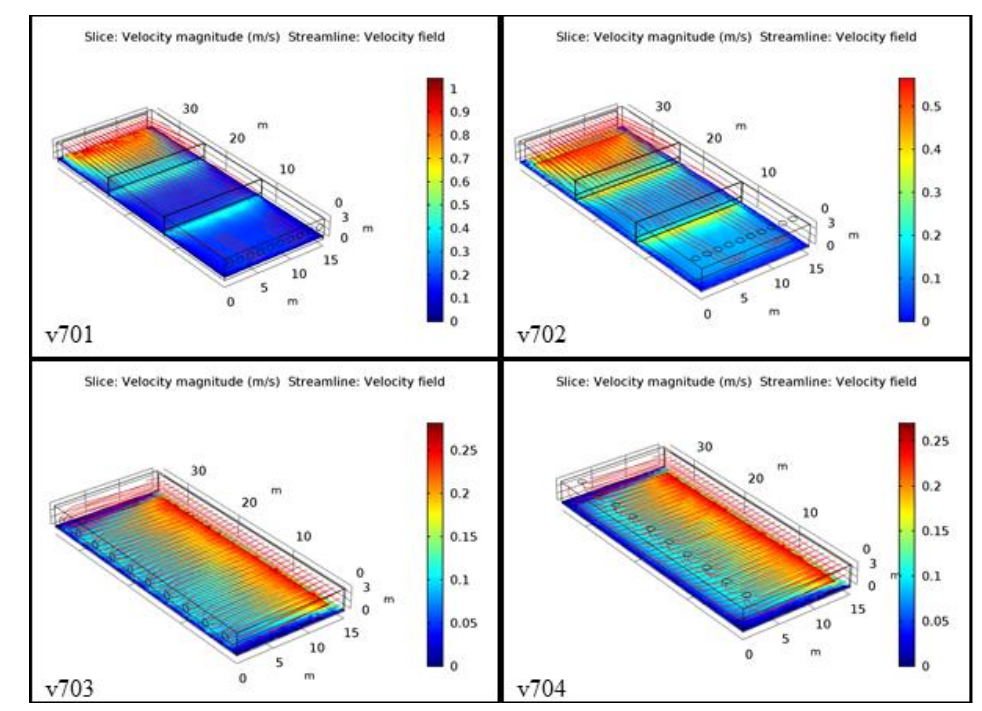

Fig. 4 - Perfil velocidade na $\boldsymbol{R A}$

A concentração de $\mathrm{NH}_{3}$ está de acordo com o perfil velocidade - temperatura, em áreas onde o ar permanece estagnado, há geração de calor, consequentemente de gases. Em todos os ambientes simulados os resultados obtidos permaneceram dentro dos parâmetros estabelecidos. Quando comparado o arranjo físico adotado, nos aviários v703 e v704 os resultados foram menores devido à homogeneidade da distribuição do fluxo de ar e a facilidade de renovação de ar. A posição dos ventiladores nos arranjos adotados explica pontos onde a concentração do gás permaneceu mais alta. 


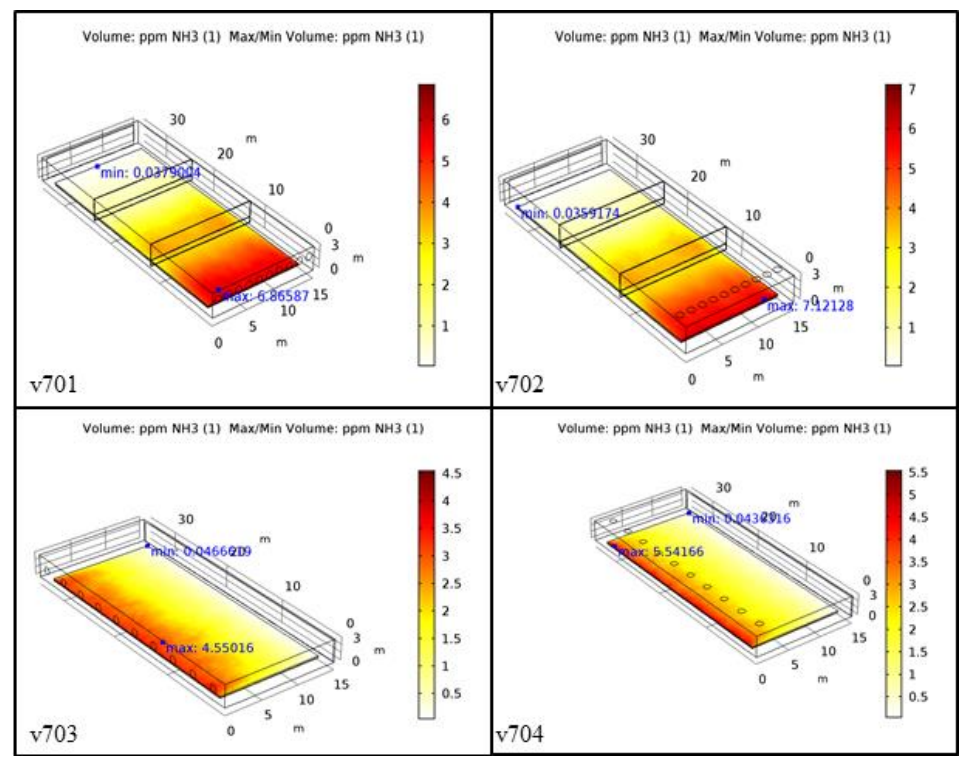

Fig. 5 - Concentração de $\mathrm{NH}_{3}$ na $\boldsymbol{R} \boldsymbol{A}$

\section{CONCLUSÃO}

As simulações confirmaram sua importância como ferramenta indispensável para projeto, análise e dimensionamento dos aviários. A modelagem utilizada provou ser fundamental para a descrição do comportamento fluidodinâmico e térmico da região das aves. $\mathrm{O}$ posicionamento dos ventiladores e a escolha do arranjo físico adequado mostraram - se um parâmetro decisivo para eliminação de pontos quentes e áreas estagnadas no interior do aviário. Os aviários simulados mostraram - se favoráveis ao crescimento e a saúde das aves.

\section{REFERÊNCIAS}

1. BATISTA, M. Simulação de um aviário: Uma análise das condições de conforto para as aves. Monografia (Graduação em Engenharia Química) Faculdade de Telêmaco Borba. Telêmaco Boorba, PR, 81 p., 2016.

2. AVILA, Valdir Silveira de,Helenice Mazzuco, Elsio Antônio Pereira de Figueiredo, Cama de Aviário, circular técnica Nº16, Empresa Brasileira de Pesquisa Agropecuária - EMBRAPA, 1992, ISSN: 0102-3713.

3. ABREU, Paulo Giovanni; ABREU, Valéria Maria N. Os desafios da ambiência sobre os sistemas de aves no Brasil. R. Bras. Zootec., v.40, p.1-14, 2011.

4. BOAVENTURA. AGRO. Aviário pressão negativa - Isolamento e velocidade do ar. Disponível em: < http://frangosonline.blogspot.com.br/2012/03/aviario-pressaonegativa-isolamento-e.html>. Acessado em 11/06/2016.

5. CURI, THAYLA M.R.C.de. Avaliação do sistema de ventilação em instalações para frangos de corte através de diferentes modelagens. 2014. 263p. Tese (Doutorado) Universidade Estadual de Campinas. Campinas: 2014.

6. SARAZ, J. A. O.; TINÔCO, ILDA DE FÁTIMA F.; ROCHA, K.S.O.; MENDES, L. B. AND NORTON, TOMAS. A cfd based approach for determination of ammonia concentration profie and flx from poultry houses with natural ventilation, 2015. 
7. BSL, R. Byron Bird \& Warren E. Stewart \& Edwin N. Lightfoot; Transport Phenomena, Second Edition, 2002, John Wiley \& Sons, Inc., ISBN 0-471-41077-2; (alternativamente, $1^{\mathrm{a}}$ edição, 1960).

8. DEEN, William M., Analysis of transport phenomena, Oxford University Press, 2nd edition 2012, isbn 978-0-19-974028-4.

9. ZENG, Z., Grigg, R., A criterion for non-Darcy flow in porous media, Transp. Porous Med., 2006, 63, 57-69.

10. SMAN, van der, R.G.M.; Prediction of airflow through a vented box by the DarcyForchheimer equation; Journal of Food Engineering, 55 (2002) 49-57.

11. DELGADO, Frank A. Coutelieris \& J.M.P.Q.; Transport Processes in Porous Media (Series in Advanced Structured Materials), 2012, Springer, ISBN-10 $=3642279090$, ISBN-13= 978-3642279096

12. HASSANIZADEH, S.M., W.G. Gray, High velocity flow in porous media, Trans. Porous Med., 2, (1987) 521-531.

13. JAMBHEKAR, Vishal A., Forchheimer Porous-media Flow Models - Numerical Investigation and Comparison with Experimental Data, Master Thesis, Universität Stuttgart - Institut für Wasser- und Umweltsystemmodellierung, Lehrstuhl für Hydromechanik und Hydrosystemmodellierung, Stuttgart, 26. November 2011.

14. HELLSTRÖM, J. G. I., T. S. Lundström, Flow through Porous Media at Moderate Reynolds Number, 4th International Scientific Colloquium Modelling for Material Processing, edited by Laboratory for Mathematical Modelling of Environmental and Technological Processes, University of Latvia, Riga, June 8-9, 2006, isbn $9984783855,9789984783857$.

15. ENGLER, Thomas W., Fluid Flow in Porous Media, Petroleum Engineering, 2010.

16. WHITAKER, S., The Forchheimer Equation: A Theoretical Development, Transport in Porous Media 25: 27-61, 1996.

17. BIRD, R. Byron Bird \& Warren E. Stewart \& Edwin N. Lightfoot; Transport Phenomena, Second Edition, 2002, John Wiley \& Sons, Inc., ISBN 0-471-41077-2; (alternativamente, $1^{\mathrm{a}}$ edição, 1960)

18. Macdonald,I. F., M. S. El-Sayed, K. Mow, and F. A. L. Dullien, Flow through Porous Media-the Ergun Equation Revisited, Ind. Eng. Chem. Fundam., Vol. 18, No. 3, 1979, 199-208.

19. Luciano B. Mendes, et. al Ammonia emissions from a naturally and a mechanically ventilated broiler house in Brazil; R. Bras. Eng. Agríc. Ambiental, v.18, n.11, p.11791185, 2014; DOI: http://dx.doi.org/10.1590/1807-1929/agriambi.v18n11p1179-1185 